\title{
The Difficult Path to Correct Diagnosis of Hepatolithiasis: A Case Report
}

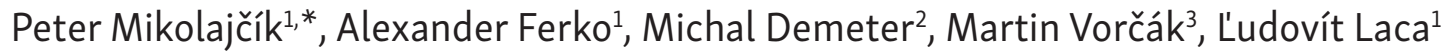

\begin{abstract}
Hepatolithiasis is a benign disease, where stones are localized proximal to the confluence of hepatic ducts. The clinical picture may differ depending on whether the stones cause complete, partial, or intermittent biliary obstruction. The course can vary from asymptomatic to fatal, thus, early diagnosis and treatment is critical for a good prognosis. The gold standard in imaging is magnetic resonance cholangiopancreatography (MRCP). However, correct diagnosis can be challenging due to atypical clinical picture and laboratory findings. We present a case where hepatolithiasis was misdiagnosed initially due to incomplete reporting and documentation of MRCP. Choledocholithiasis was diagnosed based on initial MRCP, and endoscopic stone extraction was indicated. However, an unusual postinterventional course and signs of obstructive cholangitis led to an endoscopic re-intervention, which confirmed absence of pathology in extrahepatic biliary ducts. The cholangitis recurrence required intensive antibiotic treatment, and CT examination revealed intrahepatic S3 bile duct dilatation. Thus, a re-evaluation of initial MRCP and repeated MRCP confirmed hepatolithiasis. Further, laparoscopic bisegmentectomy was chosen as the definitive treatment due to the location of the lesion. The patient recovered and remained symptom free upon a 12 month follow up.
\end{abstract}

\section{KEYWORDS}

hepatolithiasis; intrahepatic stones; choledocholithiasis; laparoscopic liver resection; laparoscopic bisegmentectomy

\section{AUTHOR AFFILIATIONS}

${ }^{1}$ Department of Surgery and Transplant Unit, Comenius University in Bratislava, Jessenius Medical Faculty in Martin, University Hospital Martin, Slovak Republic

${ }^{2}$ Department of Gastroenterology, Comenius University in Bratislava, Jessenius Medical Faculty in Martin, University Hospital Martin, Slovak Republic

${ }^{3}$ Department of Radiology, Comenius University in Bratislava, Jessenius Medical Faculty in Martin, University Hospital Martin, Slovak Republic

* Corresponding author: Department of Surgery and Transplant Unit, Comenius University in Bratislava, Jessenius Medical Faculty in Martin, University Hospital Martin, Kollárova 2, 03659 Martin, Slovak Republic; e-mail: peto.mikolajcik@gmail.com

Received: 16 January 2021

Accepted: 5 February 2021

Published online: 30 JUly 2021

Acta Medica (Hradec Králové) 2021; 64(2): 125-128

https://doi.org/10.14712/18059694.2021.21

(c) 2021 The Authors. This is an open-access article distributed under the terms of the Creative Commons Attribution License (http://creativecommons.org/licenses/by/4.0), which permits unrestricted use, distribution, and reproduction in any medium, provided the original author and source are credited. 


\section{INTRODUCTION}

Hepatolithiasis is the presence of stones proximal to the confluence of right and left hepatic duct. The symptomatology depends on the stage and severity of the obstruction and can vary from the asymptomatic course to cholangitis with fulminant sepsis. Typical clinical manifestations are cholangitis of varying intensity and jaundice (1). The treatment options are not uniform and despite treatment, hepatolithiasis often recurs. Recurrent inflammation of the bile ducts results in their fibrosis and stenosis, abscesses formation, cirrhosis, or liver fibrosis. In the long term, there is also an increased risk of developing cholangiocellular carcinoma, which occurs in $3.3 \%$ to $10 \%$ of patients with hepatolithiasis (2). Genetic predisposition, bile stasis, recurrent cholangitis, and biliary parasites are considered risk factors for hepatolithiasis $(3,4)$.

\section{CASE REPORT}

A 44-year-old male patient was referred to our hospital in August 2019 with a diagnosis of choledocholithiasis, which had been verified by magnetic resonance cholangiopancreatography (MRCP). Only a description, and no image documentation, of the procedure was available. Apart from choledocholithiasis, no other pathology was described. The patient had undergone cholecystectomy due to biliary colic 11 years ago. However, during the recent visit, he reported radiating colic abdominal pain below the right rib arch and anorexia associated with weight loss. There were no signs of jaundice or fever. Laboratory findings indicated a slight increase in gamma glutamyl transferase (GGT; $4.77 \mu \mathrm{kat} / \mathrm{l}$, normal range 0.03-0.92 $\mu \mathrm{kat} / \mathrm{l}$ ) and alanine aminotransferase (ALT; $1.47 \mu \mathrm{kat} / \mathrm{l}$, normal range $0.1-0.85 \mu \mathrm{kat} / \mathrm{l})$ serum levels. Both total and conjugated bilirubin, alkaline phosphatase (ALP), and aspartate aminotransferase (AST) levels were all within normal ranges.

The patient was admitted for endoscopic retrograde cholangiopancreatography (ERCP). During the examination, a short stump of the ductus cysticus was observed, and the hepatocholedochus was reported to be $7 \mathrm{~mm}$ wide. Above the papilla of Vater, the defect in filling of contrast revealed a choledocholite, $7 \mathrm{~mm}$ in diameter (Fig. 1). Other findings did not indicate apparent pathology. Extraction of choledocholite and lavage of bile ducts was performed. Postoperatively, there was an elevation in AST ( $2.85 \mu \mathrm{kat} / \mathrm{l}$, normal range 0.1-0.85 $\mu \mathrm{kat} / \mathrm{l})$, ALT (4.47 $\mu \mathrm{kat} / \mathrm{l})$, and GGT $(5,99 \mu \mathrm{kat} / \mathrm{l})$ levels; however, serum amylase levels did not increase. This observation was interpreted incorrectly and the patient was discharged.

Two weeks after initial ERCP, the patient returned with complaints of severe abdominal pain in the epigastrium, fever, and chills. Elevation of inflammatory (leukocytosis $13.3 \times 10^{9} / 1$, normal range 3.9-10 × 10\% $/$; CRP $27.7 \mathrm{mg} / 1$, normal range $0-5 \mathrm{mg} / \mathrm{l}$ ) and hepatic parameters (total bilirubin $31.5 \mu \mathrm{mol} / \mathrm{l}$, normal range 5-21 $\mu \mathrm{mol} / \mathrm{l}$; ALT $2.42 \mu \mathrm{kat} / \mathrm{l}$; AST $1.74 \mu \mathrm{kat} / \mathrm{l}$; GGT $2.89 \mu \mathrm{kat} / \mathrm{l}$ ) were detected in the laboratory findings. Serum amylase and ALP levels were normal. The patient was admitted and treated empirically

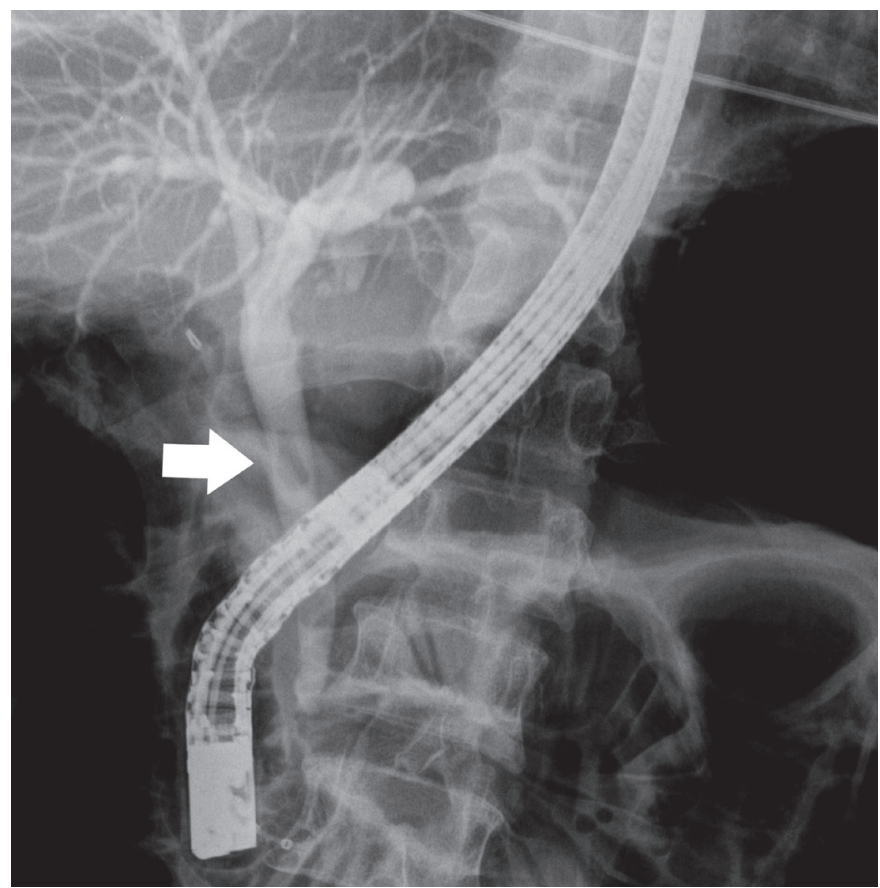

Fig. 1 Initial endoscopic retrograde cholangiopancreatography findings. Defect in the filling of contrast in the suprapapillar region - choledocholite (arrow). Other findings were without apparent pathology.

with antibiotics. Contrast-enhanced computed tomography (CT) showed dilation of bile ducts in the segment 3 (S3) of the liver to up to $10 \mathrm{~mm}$ (Fig. 2A). The patient was indicated for re-ERCP, where no pathology was found in the extrahepatic bile ducts, or in the right and left hepatic ducts. However, amputation of the subsegmental branch of the bile duct for S3 was observed (Fig. 2B). Cholangitis was managed conservatively; the patient was discharged and indicated for control MRCP one month apart.

On control MRCP, dilation of bile ducts was observed in segment 3 of the liver, without unambiguous presence of tumor (Fig. 3A). Subsequently, the finding of the initial MRCP was revisited. Image documentation, which was not available at the first examination, was requested. The finding of pathology in the S3 was already evident on the initial MRCP but was overlooked and not described in the written report (Fig. 3B). Due to the fact that the patient had a recurrence of biliary symptoms and it was not possible to rule out malignancy with certainty (despite the normal levels of tumor markers CA 19.9 and AFP), laparoscopic revision was finally preferred before further diagnostics. During the procedure, macroscopic changes were visible in the S3 of the liver (Fig. 4A) and perioperative ultrasonography confirmed the finding of dilation of the bile ducts with bile stones (Fig. 4B). Subsequently, the laparoscopic bi-segmentectomy S2-S3 was performed. The operative and postoperative course were without complications and the patient was discharged on the 4 th postoperative day. Histological examination confirmed hepatolithiasis and ruled out malignancy (Fig. 4C). No residual hepatolithiasis was present in the control MRCP performed 3 months post-surgery. The patient had recovered, with no recurrence of biliary symptoms when followed-up for more than 12 months after the operation. 
A

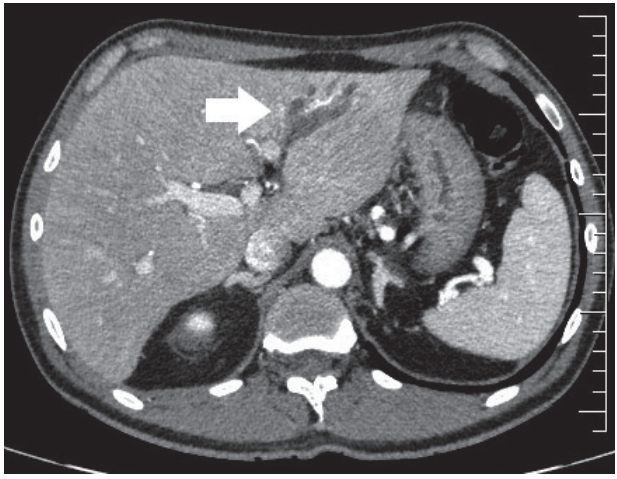

B

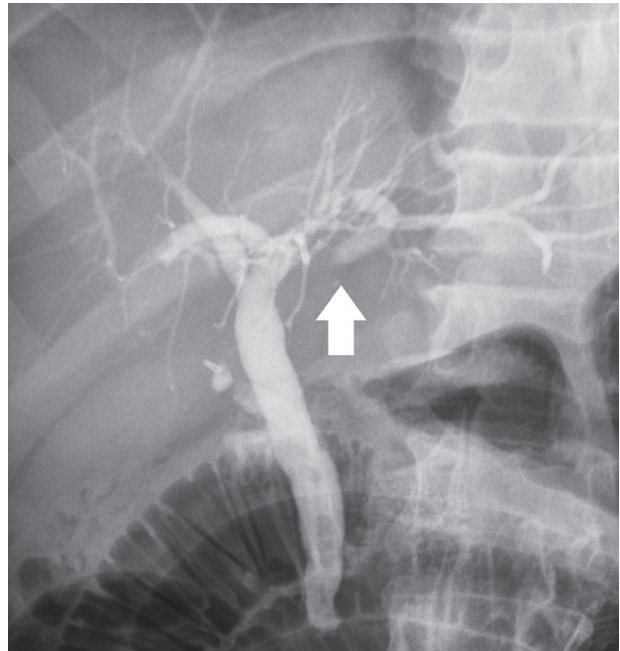

Fig. 2 Findings of imaging examinations during the first rehospitalisation. (A) Contrast-enhanced computed tomography (CT) scan shows dilatation of the bile ducts in segment 3 of the liver up to $10 \mathrm{~mm}$, there is also higher post-contrast enhancement of the bile ducts walls and the surrounding liver parenchyma in this area (arrow) - CT signs of cholangitis; (B) re-endoscopic retrograde cholangiopancreatography examination without finding pathology on the extrahepatic bile ducts and on the right and left hepatic duct. There is visible amputation of the subsegmental branch of the bile duct in segment 3 of the liver (arrow).

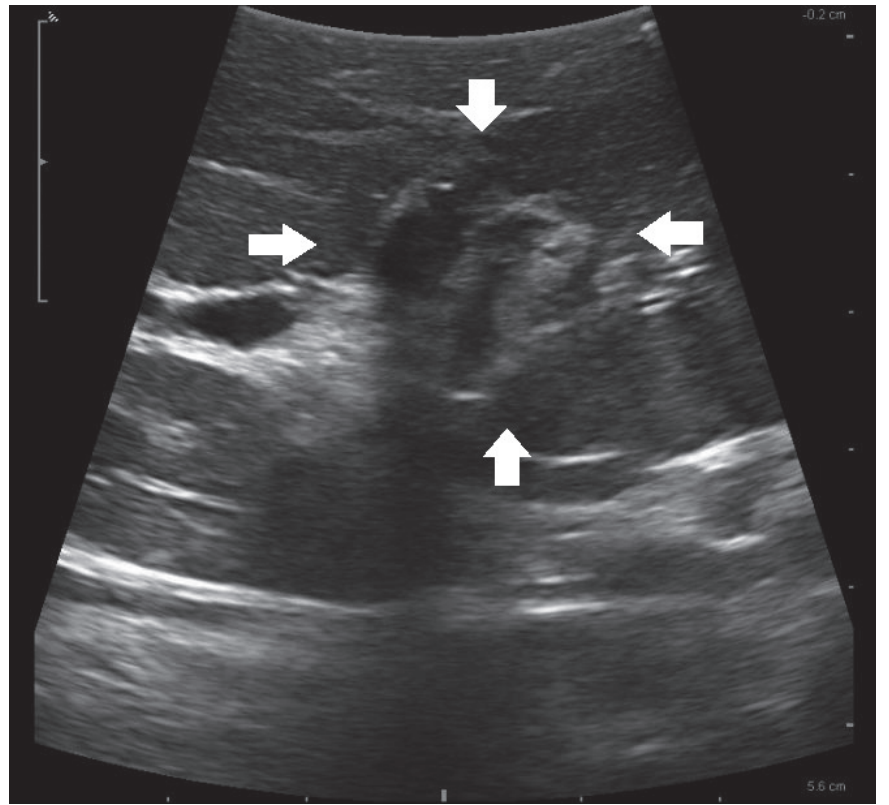

A

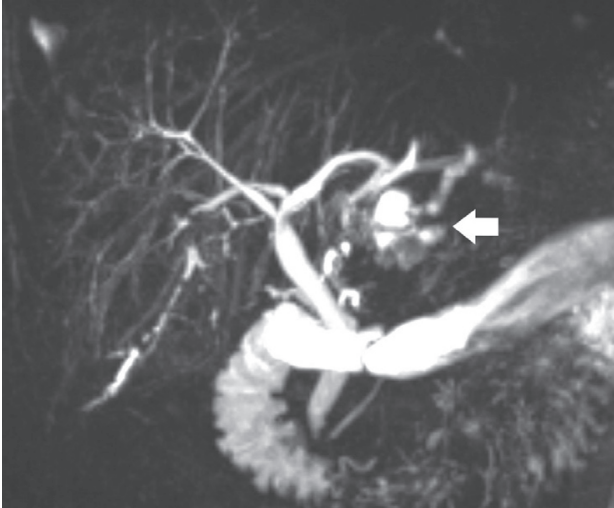

B

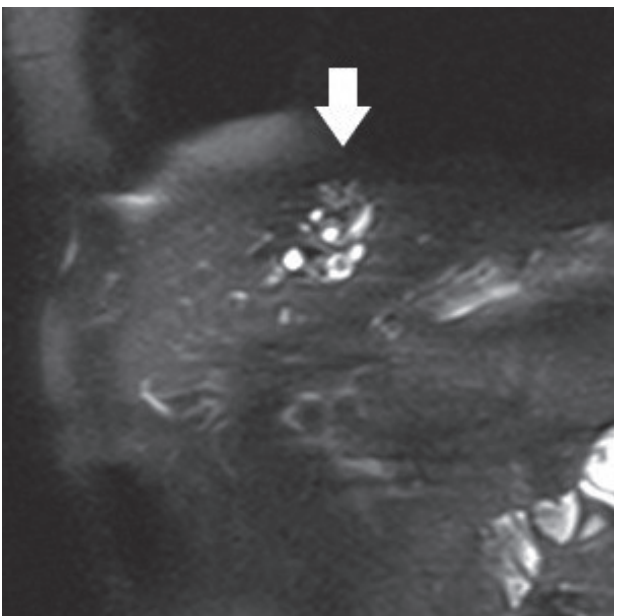

Fig. 3 Comparison of magnetic resonance

cholangiopancreatography (MRCP) findings. (A) Control MRCP shows bile duct dilatation in the segment 3 of the liver (arrow); (B) Initial MRCP reveals apparent dilatation of bile ducts in the segment 3 of the liver at time of initial diagnosis (arrow).
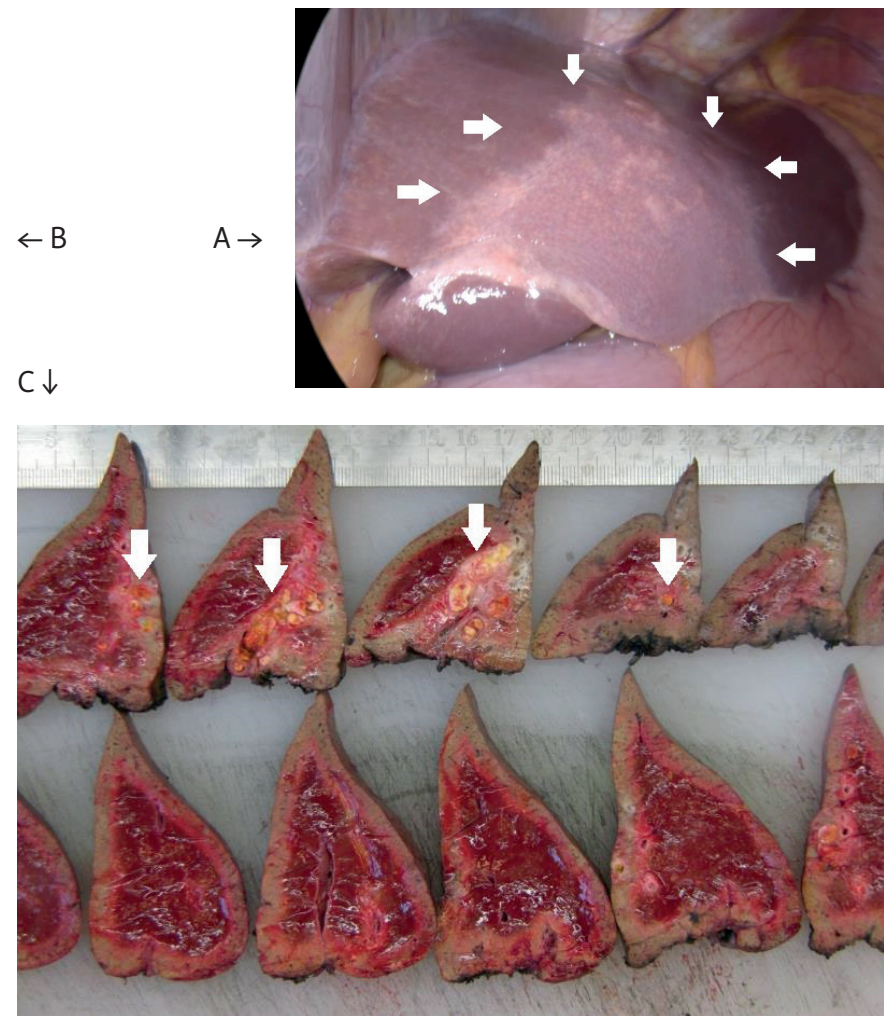

Fig. 4 Intraoperative and histopathological findings. (A) Macroscopic changes in the segment 3 of the liver during laparoscopic revision (arrows); (B) Intraoperative sonography revealed the dilatation of ducts and bile stones (arrows); (C) The specimen of the segments 2 and 3 of the liver, a large ductus filled with yellow stones is visible in serial sections (arrows). 


\section{DISCUSSION}

Hepatolithiasis is a benign disease, but its symptoms of biliary obstruction and cholangitis can be fatal within 24 hours due to fulminant sepsis $(1,4)$. Early diagnosis and aggressive treatment are key to a good prognosis. In the case of extensive hepatolithiasis and recurrent cholangitis, cirrhosis, fibrosis, and eventually liver failure may occur. Another risk is development of cholangiocellular carcinoma $(2,4)$. The incidence of hepatolithiasis in the indigenous people of the Western countries remains very low; therefore, this diagnosis is not immediately considered in patients with biliary symptoms (5).

Clinical symptomatology depends mainly on the severity and location of biliary tract obstruction $(1,6)$. In our patient, especially during the primary examination, the typical clinical symptomatology was not fully plotted and the biliary symptoms were attributed to choledocholithiasis. Even during further course of the disease development to cholangitis, although abdominal pain was associated with chills and fever, jaundice was not clinically present.

In the case of obstruction and cholangitis, typically the markers of bile duct obstruction, hepatocyte damage, and inflammation are elevated. However, in case of incomplete obstruction, early after the onset of symptoms and when the hepatolithiasis is located in more peripheral ducts, laboratory finding may not be typical (6). In our patient, only some markers of obstruction and hepatocyte damage were found slightly elevated in the laboratory findings during the primary examination. This was probably due to incomplete obstruction without cholangitis. After the ERCP extraction of choledocholite, elevation of hepatic parameters occurred, but due to the description of initial MRCP and safe removal of choledocholite, this observation was misinterpreted and the patient was discharged. During rehospitalisation, the laboratory findings were almost typical of cholangitis.

Sonography and contrast-enhanced CT may contribute to the diagnosis of hepatolithiasis. However, the gold standard in the imaging of hepatolithiasis is MRCP (7). ERCP is usually indicated either as a predominantly therapeutic method, only after the bile duct obstruction is diagnosed, or in case of diagnostic doubts. In the case of our patient, the initial MRCP would have, upon careful evaluation, revealed hepatolithiasis in addition to choledocholithiasis. Relying on the description of MRCP without looking at the image documentation, the rarity of hepatolithiasis in our region, and the coincidence with choledocholithiasis were factors that influenced our decision-making and led to a delay in making a definitive diagnosis of hepatolithiasis. As in this case, ERCP is the method of choice for the treatment of choledocholithiasis. Acute contrast-enhanced CT revealed dilatation of the bile ducts in segment 3 of the liver and signs of cholangitis in this area. Subsequent re-ERCP revealed amputation of the subsegmental branch of the bile duct for S3, which could not be treated endoscopically due to its unavailability. Control MRCP led to a definitive diagnosis of hepatolithiasis. This case highlights the fact that the currently available laboratory and imaging techniques are able to establish a definitive diagnosis of hepatolithiasis, if properly examined and interpreted.

The biggest challenge, therefore, remains the adequate and effective treatment that allows for restoration of bile drainage by removing the bile stones or strictures and, if the parenchyma is damaged, its resection (3). Choice of treatment depends on the extent of hepatolithiasis (5, 8). Liver resection is considered as the optimal treatment because it removes damaged bile ducts and parenchyma, thereby minimizing the risk of recurrence of hepatolithiasis and cholangiocellular carcinoma formation $(3,9)$. Vetrone reports that up to $4.5 \%$ of liver specimens resected for hepatolithiasis have been shown by histological examination to have previously undiagnosed cholangiocellular carcinoma (9). However, resection cannot be performed in patients with extensive hepatolithiasis $(3,9)$. In the case of our patient, hepatolithiasis was localized only in segment 3 of the liver. Therefore, resection was chosen as the optimal therapy in this case as well. The operation and postoperative course were without complications and the patients biliary symptoms did not recur. Histological examination definitively ruled out the presence of cholangiocellular carcinoma. Our experience confirms the suitability of liver resection as the optimal and most effective solution in cases of localized hepatolithiasis.

In conclusion, the course of hepatolithiasis can vary from asymptomatic to fatal and also laboratory finding may not be typical. MRCP is the gold standard in imaging of hepatolithiasis. Nowadays, if currently available laboratory and imaging techniques are properly examined and interpreted, there should not be problem to establish a definitive diagnosis of hepatolithiasis. Also it is very important, that early diagnosis and treatment is crucial for a patient's prognosis. Choice of treatment mainly depends on the extent of hepatolithiasis and in cases of localized hepatolithiasis, liver resection is considered as the optimal treatment.

\section{DISCLOSURES}

Drs. Peter Mikolajčík, Alexander Ferko, Michal Demeter, Martin Vorčák and Ludovít Laca have no conflicts of interest or financial ties to disclose.

\section{REFERENCES}

1. Huang M. Long-term outcome of percutaneous transhepatic cholangioscopic lithotomy for hepatolithiasis. Am J Gastroenterol 2003; 98(12): 2655-62.

2. Liu ZY, Zhou YM, Shi LH, Yin ZF. Risk factors of intrahepatic cholangiocarcinoma in patients with hepatolithiasis: a case-control study. Hepatobiliary Pancreat Dis Int 2011; 10(10): 626-31.

3. Wen XD, Wang T, Huang Z, et al. Step-by-step strategy in the management of residual hepatolithiasis using post-operative cholangioscopy. Ther Adv Gastroenterol 2017; 10(11): 853-64.

4. Tazuma S. Epidemiology, pathogenesis, and classification of biliary stones common bile duct and intrahepatic). Best Pract Res ClinGastroenterol 2006; 20(6): 1075-83.

5. Lorio E, Patel P, Rosenkranz L, Patel S, Sayana H. Management of Hepatolithiasis: Review of the Literature. Curr Gastroenterol Rep 2020; 22(6): No of issue 30.

6. Bonheur JL, Ells PF. Biliary obstruction. WebMD LLC. 2021. (Updated 16/10/2019) (Accessed January 3, 2021, at https://emedicine .medscape.com/article/187001).

7. Park DH, Kim MH, Lee SS, et al. Usefulness and limitation of magnetic resonance cholangiopancreatography in patients with hepatolithiasis. Korean J Gastroenterol 2003; 42(5): 423-30

8. Tsuyuguchi T, Miyakawa K, Sugiyama H, et al. Ten-year long-term results after non-surgical management of hepatolithiasis, including cases with choledochoenterostomy. J Hepatobiliary Pancreat Sci 2014; 21 (11): 795-800.

9. Vetrone G, Ercolani G, Grazi, G, et al. Surgical Therapy for Hepatolithiasis: A Western Experience. J Am Coll Surg 2006; 202(2): 306-12. 